\title{
Recruitment Strategies and the Retention of Obese Urban Racial/Ethnic Minority Adolescents in Clinical Trials: The FIT Families Project, Michigan, 2010-2014
}

\author{
Kathryn Brogan Hartlieb, PhD, RD; Angela J. Jacques-Tiura, PhD; Sylvie Naar-King, PhD; \\ Deborah A. Ellis, PhD; Kai-Lin Catherine Jen, PhD; Sharon Marshall, MD
}

\begin{abstract}
Suggested citation for this article: Hartlieb KB, Jacques-Tiura AJ, Naar-King S, Ellis DA, Jen KC, Marshall S. Recruitment Strategies and the Retention of Obese Urban Racial/Ethnic Minority Adolescents in Clinical Trials: The FIT Families Project, Michigan, 2010-2014. Prev Chronic Dis 2015;12:140409. DOI: http://dx.doi.org/10.5888/pcd12.140409.
\end{abstract}

\section{PEER REVIEWED}

\section{Abstract}

\section{Introduction}

The successful recruitment and retention of participants is integral to the translation of research findings. We examined the recruitment and retention rates of racial/ethnic minority adolescents at a center involved in the National Institutes of Health Obesity Research for Behavioral Intervention Trials (ORBIT) initiative by the 3 recruitment strategies used: clinic, informatics, and community.

\section{Methods}

During the 9-month study, 186 family dyads, each composed of an obese African American adolescent and a caregiver, enrolled in a 6-month weight-loss intervention, a sequential multiple assignment randomized trial. We compared recruitment and retention rates by recruitment strategy and examined whether recruitment strategy was related to dyad baseline characteristics.

\section{Results}

Of the 186 enrolled families, $110(59.1 \%)$ were recruited through clinics, $53(28.5 \%)$ through informatics, and $23(12.4 \%)$ through community. Of those recruited through community, $40.4 \%$ enrolled in the study, compared with $32.7 \%$ through clinics and $8.2 \%$ through informatics. Active refusal rate was 3\%. Of the 1,036 families identified for the study, 402 passively refused to participate: 290 (45.1\%) identified through informatics, 17 (29.8\%) through community, and 95 (28.3\%) through clinics. Recruitment strategy was not related to the age of the adolescent, adolescent comorbidities, body mass index of the adolescent or caregiver, income or education of the caregiver, or retention rates at 3 months, 7 months, or 9 months. Study retention rate was $87.8 \%$.

\section{Conclusion}

Using multiple recruitment strategies is beneficial when working with racial/ethnic minority adolescents, and each strategy can yield good retention. Research affiliated with health care systems would benefit from the continued specification, refinement, and dissemination of these strategies.

\section{Introduction}

Excessive body weight is one of the most prevalent medical problems among children and adolescents despite significant attention and funding (1-3). According to 2011-2012 data from the National Health and Nutrition Examination Survey, 39.8\% of non-Hispanic black adolescents (aged 12-19) are overweight or obese, compared with $31.2 \%$ of non-Hispanic white adolescents (4). Thus the study of weight-loss treatments for adolescents, particularly racial/ethnic minority adolescents, is an important research focus.

The ability to effectively recruit and retain racial/ethnic minority adolescents and their families in research is imperative. Strategies for recruiting and retaining minority research participants emphasize community involvement, convenience of meeting times and locations, and rapport with research staff (5-8). Strategies for recruiting and retaining racial/ethnic minority adolescents for research mirror those recommended for nonminority participants (9-11), with the addition of extensive follow-up (12). Because of 
the demands of extensive follow-up, successful recruitment and retention of minority adolescents and their families may require substantial time and resources of research staff.

Weight-loss trials among minority adolescents have traditionally used community-based recruitment methods $(13,14)$, such as radio advertisements, or clinic-based methods (15), such as provider referrals. However, clinical informatics - the application of information technology (eg, screening for eligible participants using electronic medical records [EMRs]) - can increase the quality and efficiency of clinic-based methods by incorporating the processes and resources of the biomedical sector $(16,17)$. Informatics, when used in addition to traditional recruitment strategies, can improve enrollment by enhancing identification of and access to participants.

The objectives of this study were to 1) describe the enrollment of obese racial/ethnic minority adolescents in a 6-month weight-loss intervention (FIT Families) using 3 recruitment strategies (clinic, informatics, community), 2) compare the 3-month, 7-month, and 9-month retention rates of the 3 strategies, and 3) identify baseline participant characteristics that may be associated with retention rates.

\section{Methods}

\section{Study design}

Our center, the Wayne State University Pediatric Weight Management Center, as a partner in the National Institutes of Health's initiative, Obesity Research for Behavioral Intervention Trials (ORBIT), brought together a multidisciplinary research group composed of 1) obesity intervention researchers with extensive experience in adolescent health behavior change, 2) basic behavioral scientists with experience in motivation and learning, 3) registered dietitians and nutritionists with expertise in dietary and weightloss interventions, and 4) communication scientists who focus on interactions between health providers and families in urban populations. The goal of the center was to develop an adaptive weightloss treatment for obese African American adolescents. Establishing successful recruitment approaches to allow for maximum retention was a vital component.

FIT Families was a 6-month sequential multiple assignment randomized trial (SMART) (18) focusing on weight loss among obese African American adolescents (19). Recruitment began in November 2010, the first data collection took place in January 2011, and the last data collection took place in March 2014. The goal of using the SMART approach was to develop an evidence-based adaptive intervention that would be evaluated in a subsequent randomized controlled trial (20). Our SMART design had 2 random- ization points (Figure). Each study arm included an intervention contact twice per week (except for the maintenance stage, which consisted 1 session per week). Each participating family received $\$ 50$ for completion of data collection at 3 points (baseline, 7 months, and 9 months) and $\$ 10$ for completion of data collection at 3 months. Details on the intervention are described elsewhere (19).

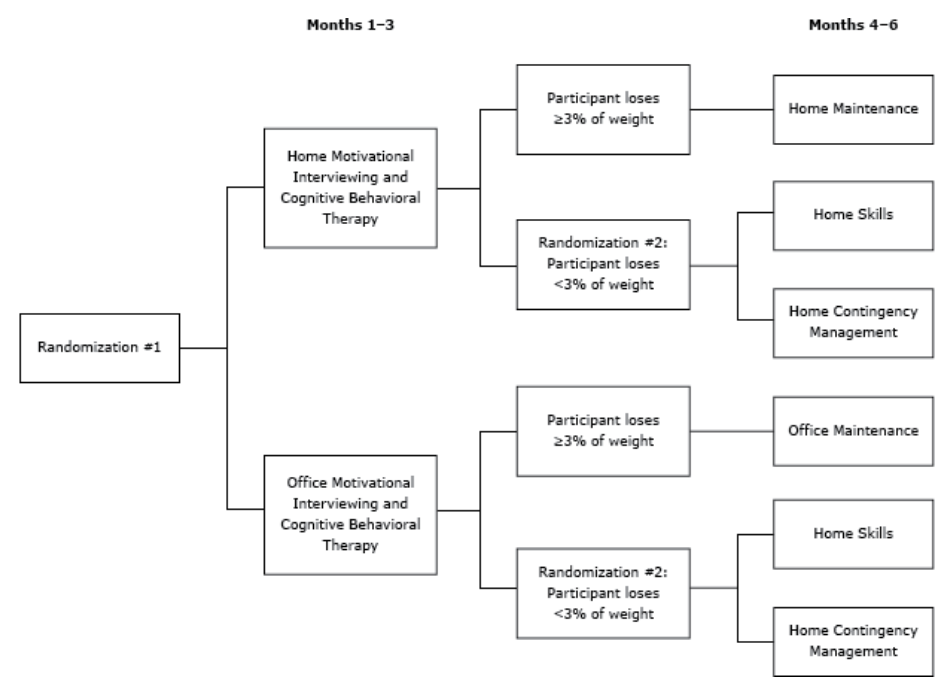

Figure. Overview of sequential multiple assignment randomized trial (SMART) design (19) for the FIT Families study, Michigan, 2010-2014. The first randomization took place at baseline and the second at 3 months. The second randomization took place if the adolescent participant did not lose $3 \%$ of his or her initial body weight. "Office" refers to the Wayne State University Pediatric Weight Management Center.

\section{Eligibility criteria}

Study inclusion criteria for the adolescent were 1) self-identified as African American, 2) aged from 12 years, 0 months through 16 years, 11 months at time of consent, 3 ) body mass index (BMI) for age in the 95th percentile or more, 4) resides 30 miles or less from study offices, 5) resides with the primary caregiver, 6) primary caregiver willing to participate in treatment, and 7) English speaking. Adolescents were excluded for the following reasons: 1) obesity was secondary to medication (eg, steroids, antipsychotics) or a chronic health condition (eg, Prader-Willi Syndrome); 2) pregnancy, 3) medical conditions for which weight loss was contraindicated, 4) thought disorder, or 5) serious cognitive impairments. For each adolescent participant, we recruited a primary caregiver so that our primary unit of analysis was a family dyad. Eligibility criteria for the caregiver were being at least 18 years old; being the legal guardian or, if not the legal guardian, having the consent of the legal guardian; and willingness to participate in treatment. Research protocols were approved by the Wayne State

\footnotetext{
The opinions expressed by authors contributing to this journal do not necessarily reflect the opinions of the U.S. Department of Health and Human Services, the Public Health Service, the Centers for Disease Control and Prevention, or the authors' affiliated institutions.
} 
University Human Investigation Committee, and investigators reported outcomes every 6 months to a data safety and monitoring board.

\section{Recruitment strategies}

\section{Clinics}

Clinic-based recruitment took place in a large urban children's hospital that serves 60,000 children annually. This strategy involved direct collaboration with clinic health care providers. A 15minute orientation was held with outpatient health care providers from various disciplines (eg, adolescent medicine, ambulatory pediatrics, asthma, diabetes); the session emphasized the importance of study eligibility criteria. Health care providers received a study binder that contained information on eligibility criteria, scripts for introducing the study and talking about it to potential family participants, and brochures to support a 1-minute introduction (including research staff contact information) of the study to families. Families interested in learning more about the study were asked to complete a release-of-information form so that research staff could follow up with further information. Research staff spent approximately 1 hour each week visiting clinics to collect forms and provide a visual reminder of the study to health care providers. A nurse manager was the main contact in clinics with large numbers of rotating residents (eg, ambulatory pediatrics); an attending physician was the main contact in specialty clinics (eg, diabetes). Clinic providers expressed appreciation for the option of offering a weight-management resource to patients and did not request additional compensation. This recruitment strategy allowed the research team to recruit from multiple clinics while making minimal demands on the time of clinical and research staff.

\section{Informatics}

Unlike younger children, adolescents often do not have medical appointments for regular well-child check-ups $(21,22)$; this lack of regular medical checkups limits researchers' ability to recruit adolescents through clinics. Therefore, we added the use of informatics as a second recruitment strategy. Although BMI data in the EMRs at some of the clinics offered the potential to identify eligible adolescents, we could not use BMI percentile as a search criterion in the overall hospital EMR system. Instead, we relied on medical billing information in the EMR for data on obesity. Informatics identified adolescents who were seen as inpatients, in outpatient clinics, or in the emergency department for whom medical billing included an International Classification of Diseases, Ninth Revision code for obesity, acanthosis nigricans, metabolic syndrome, or type 2 diabetes. A recruitment letter and a study brochure were mailed to potentially eligible families from the hospit- al's adolescent medicine division. The letter stated that the family had 2 weeks to opt out of being contacted for the study; otherwise a FIT Families research assistant would telephone to provide more information.

\section{Community}

Community-based recruitment consisted of free postings (eg, flyers on bulletin boards, brochures) at institutions serving racial/ethnic minority populations and media announcements through the university and hospital. Research assistants offering nutrition and study information attended 5 health fairs at local schools and churches. This recruitment strategy also included referrals through word-of-mouth by enrolled participants to others in the community.

\section{Screening and enrollment}

Participant screening and enrollment involved a 3-step EMRbased, telephone-based, and home-based process. Research staff spent 1.5 hours per week reviewing EMR data to exclude adolescents who did not meet eligibility criteria. EMR screening often provided eligibility information on adolescent race/ethnicity, height and weight (for calculation of BMI), age, and distance of residence from study offices. Telephone screening was completed with the adolescent's primary caregiver. The time spent on the telephone with each family recruited through the clinic-based and community-based strategies typically was 20 minutes, whereas research assistants typically spent an additional 5 to 10 minutes on the telephone with each family recruited through the informatics strategy because these families had less knowledge of the study. Research staff initially attempted to contact families twice weekly following a standardized structure of different times (morning, midday, afternoon, evening) on different days of the week and weekend. Brief messages with call-back information were left on machines (when possible) or with people other than the primary caregiver; confidentiality was maintained. When a family could not be reached after 1 month, research staff called the family twice per month. Once a family was reached, the telephone screen entailed a series of questions to establish eligibility. A family was counted as a passive refusals or lost contact if the research staff could not reach it for any reason (eg, telephone messages were disregarded, contact information was incorrect). If eligibility was established by the telephone screen, the research assistant scheduled a home-based screening and consent visit. After research staff confirmed eligibility in person during the home visit, which included a screening for the potential participant's level of safety in engaging in physical activity, the researcher obtained informed consent from the caregivers and assent from the adolescents. Because of the complex SMART study design, extra attention was paid to explain the 6 intervention arms to study participants (19).

The opinions expressed by authors contributing to this journal do not necessarily reflect the opinions of the U.S. Department of Health and Human Services, the Public Health Service, the Centers for Disease Control and Prevention, or the authors' affiliated institutions. 
Differences between compensation for data collection and intervention visits were also explained. The home visit took 30 to 45 minutes. After providing consent, families completed the 2-hour data collection process; which included questionnaires and anthropometric measurements conducted by the research assistants. For this report, we collected data on the following characteristics: height and weight of the adolescent and the caregiver (to calculate $\mathrm{BMI}$ as weight in $\mathrm{kg}$ divided by height in meters square); age of the adolescent, any comorbidities of the adolescent, annual income of caregiver, and education of caregiver.

\section{Retention}

To retain families for the data collection at 3 months, 7 months, and 9 months, a reminder postcard was sent to families 4 weeks before data collection began. Telephone calls to schedule data collection appointments started 3 weeks before data collection. If a family was within 1 week of data collection and had not yet confirmed an appointment with the research staff, more intensive efforts began. These included more frequent telephone calls, home visits, or reaching out to the alternate contact people identified by the caregiver during the consent process.

\section{Staff training}

Staff training for recruitment, enrollment, and retention involved role playing and observation to ensure the research staff's fidelity to protocol. Scripts were developed for research staff to emphasize a person-centered, nondirective communication style using open questions and reflections. Quality assurance checks were conducted every 6 months by a supervisor who observed the screening and consent and assent and data collection processes. Research staff and investigators discussed recruitment and retention efforts during weekly meetings. The equivalent of 2.25 fulltime research assistants was employed for 37.5 hours per week during the study period. The 0.25 full-time research assistant helped during home-based data collection.

\section{Analytic plan}

We examined the number and percentage of families recruited from each of the 3 recruitment strategies and used $\chi^{2}$ contingency tables to determine whether any strategy was associated with study enrollment or retention at 3 months, 7 months, or 9 months. We used analysis of variance (ANOVA) and Tukey's honestly significant difference (to account for multiple comparisons) post hoc tests to determine whether any strategy was associated with the age or BMI of the adolescent or BMI of the caregiver (all continuous variables), and we used a $\chi^{2}$ test to determine whether any recruitment strategy was associated with the presence of adolescent comorbidities, caregiver education, or caregiver income (categor- ical variables). We calculated an overall enrollment rate (number of families enrolled divided by the number originally identified), and active refusal rate (number of families who actively refused divided by the number originally identified), a passive refusal rate (number of passive refusals or lost contacts divided by the number originally identified), a modified active refusal rate (number of active refusals divided by the number who passed the EMR screen), and a modified passive refusal rate (number of passive refusals or lost contacts divided by the number who passed the EMR screen).

\section{Results}

\section{Recruitment}

Of the 1,036 families identified through clinics, informatics, and community, only $30(2.9 \%)$ actively refused to participate and all who refused did so during the telephone screening (Table 1). However, 402 families passively refused or were lost contacts. Overall passive refusal rate was related to recruitment strategy $\left(\chi_{2}^{2}\right.$ $=28.4, P<.001$ ), with a larger percentage of families passively refusing who were identified through informatics $(45.1 \%)$ than through clinic $(28.3 \%)$ or community strategies (29.8\%). Among families who passed the EMR and other prescreening $(n=679)$, the modified passive refusal rate was related to recruitment strategy $\left(\chi_{2}^{2}=92.5, P<.001\right)$, with a larger percentage of families passively refusing who were identified through informatics $(74.9 \%)$ than through clinics $(39.1 \%)$ or the community $(34.7 \%)$. Of families who consented to participate $(\mathrm{n}=197)$ but did not enroll, $7.0 \%$ were recruited through informatics and $6.0 \%$ through clinics; all families recruited through the community consented and enrolled $\left(\chi_{2}^{2}=1.6, P=.44\right)$. Of the 186 enrolled families, $28.5 \%$ were identified through informatics, $59.1 \%$ through clinics, and $12.4 \%$ through the community. Enrollment rate and recruitment strategy were significantly related $\left(\chi_{2}^{2}=110.4, P<.001\right)$. Although the fewest number of families were identified through the community $(n=23), 40.4 \%$ enrolled, compared with $8.2 \%$ through informatics and $32.7 \%$ through clinics.

\section{Retention}

Five families were removed from the study by the principal investigator, 2 because of interventionist error and 3 because of discovery of ineligibility after the study began; 1 family was recruited through informatics, 1 from the community, and 3 recruited from clinics. Thus, 181 families were expected to complete all aspects of the study. No recruitment strategy was related to retention rates at 3 months $\left(\chi_{2}^{2}=1.9, P=.39\right), 7$ months, $\left(\chi_{2}^{2}=1.9, P=.38\right)$, or 9 months $\left(\chi_{2}^{2}=1.8, P=.41\right)$ (Table 2$)$. Overall study retention was $87.8 \%$.

\footnotetext{
The opinions expressed by authors contributing to this journal do not necessarily reflect the opinions of the U.S. Department of Health and Human Services, the Public Health Service, the Centers for Disease Control and Prevention, or the authors' affiliated institutions.
} 


\section{Baseline characteristics}

Recruitment strategy was not related to the BMI or age of the adolescent, presence of comorbidities, caregiver BMI, caregiver education level, or caregiver income (Table 3 ).

\section{Discussion}

The 3 recruitment strategies used in this study demonstrated processes to identify and promote research participation among obese racial/ethnic minority adolescents and their caregivers. More than half $(59.1 \%)$ of families were enrolled through the clinic-based strategy. The personal interaction with a medical clinic health care provider may have had a beneficial influence. Qualitative studies have reported positive experiences during clinician-initiated recruitment despite clinicians' initial misgivings about burdening or overwhelming families (23). A discussion about research opportunities in the context of clinical care can build patient-provider relationships regardless of research participation (24) and can thus be valuable to clinicians, patients, and researchers. Overcoming clinicians' negative feelings about approaching families for participation in research was achieved in this study through orientation sessions and well-defined recruitment procedures.

The findings of this study also indicate the value of recruitment strategies other than clinic-based recruitment. Although the proportion of families enrolled through the community $(12.4 \%)$ was the lowest of the 3 recruitment strategies, $40.4 \%$ of identified eligible participants enrolled, compared with only one-third of those identified through clinics and less than $10 \%$ through informatics. Community-based recruitment of racial/ethnic minority participants fosters trust and is a positive way to reach minority and adolescent populations $(5,25)$. Community-based recruitment was a more passive strategy (eg, health fair conversations) than the other 2 strategies but was an important avenue for linking interested and motivated participants to research.

Despite lacking the advantages of personal interaction, the informatics strategy enrolled more than one-quarter of eligible families that may otherwise have been overlooked; it is a viable approach for health care system-based research. The use of opt-out letters meant that potentially eligible families did not have to proactively indicate an intention to participate. This strategy has been found to shorten recruitment time and double the number of participants compared with an opt-in letter recruitment strategy (26). No families receiving letters in our study opted out of being contacted by research staff. The benefits of the informatics strategy may be even more pronounced during the latter years of recruitment for multiyear clinical trials when clinic and community referrals plateau.
Although our study had few active refusals (3\%) across the 3 strategies, we had many passive refusals. We were not able to screen by telephone almost three-fourths of families identified though informatics, compared with about half of families identified through clinics and one-third of families identified through the community. Overall, our findings support previous research. Racial/ethnic minority youths and families tend to participate when reached through appropriate recruitment strategies (27), and multiple strategies are required for success (28).

Because BMI data were not available in the hospital EMR system, we relied on obesity-related diagnoses for the informatics strategy. Clinicians may not submit billing for obesity unless a significant amount of excess weight is present. Therefore, during recruitment, we anticipated being unable to identify some eligible adolescents and possibly introducing bias by identifying adolescents at the upper end of the BMI scale. However, we found no differences among the populations across the 3 methods. Our recruitment strategies allowed for inclusivity of participants across BMI, age, comorbidity status, and socioeconomic status of the study location.

Our retention program targeted several key areas recommended in the literature $(5,28)$. Research staff provided accessible locations for meetings, frequent reminders, and timely incentive payments, and, for the most part, staff was consistent during the study. Overall, our study retention rate $(88 \%)$ was within the range found in similar studies $(29,30)$ and did not differ across recruitment strategies.

Our recruitment methods had limitations. Health care providers participating in the clinics did not record the number of families who were approached but refused to participate. Such data could provide important insight into the total amount of time spent by providers and research staff on recruitment. A formal time-allocation study, particularly on screening and initial contact, is warranted. Additional data are being compiled through exit interviews with study participants. An advisory board made up of community members and study participants will also be convened after completion of the study to further refine recruitment strategies.

The 3 recruitment strategies described here - clinic, informatics, and, community - led to the successful inclusion of racial/ethnic minority adolescent participants across numerous baseline characteristics. Study retention strategies were efficacious and may be practical for other research groups affiliated with health care-based systems. Research in behavioral intervention trials would benefit from the continued specification, refinement, and dissemination of these recruitment and retention strategies.

The opinions expressed by authors contributing to this journal do not necessarily reflect the opinions of the U.S. Department of Health and Human Services, the Public Health Service, the Centers for Disease Control and Prevention, or the authors' affiliated institutions. 


\section{Acknowledgments}

This study was funded by grant no. 1U01HL097889. This trial was registered at clinicaltrials.gov (registration no. NCT01350531. We thank Jessica Walker, Christina DeAngelis, and Tanya Troy for their role in this project. When this research was conducted, Dr Brogan Hartlieb was affiliated with Wayne State University.

\section{Author Information}

Corresponding Author: Kathryn Brogan Hartlieb, Department of Dietetics and Nutrition, Florida International University, Miami, FL 11200 SW 8th St, AHC-5 323, Miami, FL 33199. Telephone: 305-348-3235. Email: kabrogan@fiu.edu.

Author Affiliations: Angela J. Jacques-Tiura, Sylvie Naar-King, Deborah A. Ellis, Pediatric Prevention Research Center, Wayne State University School of Medicine, Detroit, Michigan; Kai-Lin Catherine Jen, Department of Nutrition and Food Science, Wayne State University, Detroit, Michigan; Sharon Marshall, Children's Hospital of Michigan, Wayne State University School of Medicine; Detroit, Michigan.

\section{References}

1. Khan LK, Sobush K, Keener D, Goodman K, Lowry A, Kakietek J, et al.;Centers for Disease Control and Prevention. Recommended community strategies and measurements to prevent obesity in the United States. MMWR Recomm Rep 2009;58(RR-7):1-26.

2. Let's Move. http://www.letsmove.gov/. Accessed August 1, 2014.

3. National Collaborative on Childhood Obesity Research (NCCOR). http://www.nccor.org/. Accessed August 1, 2014.

4. Ogden CL, Carroll MD, Kit BK, Flegal KM. Prevalence of childhood and adult obesity in the United States, 2011-2012. JAMA 2014;311(8):806-14.

5. Yancey AK, Ortega AN, Kumanyika SK. Effective recruitment and retention of minority research participants. Annu Rev Public Health 2006;27(1):1-28.

6. Burlew AK, Weekes JC, Montgomery L, Feaster DJ, Robbins MS, Rosa CL, et al. Conducting research with racial/ethnic minorities: methodological lessons from the NIDA Clinical Trials Network. Am J Drug Alcohol Abuse 2011; 37(5):324-32.
7. Brannon EE, Kuhl ES, Boles RE, Aylward BS, Ratcliff MB, Valenzuela JM, et al. Strategies for recruitment and retention of families from low-income, ethnic minority backgrounds in a longitudinal study of caregiver feeding and child weight. Child Health Care 2013;42(3):198-213.

8. Wallace DC, Bartlett R. Recruitment and retention of African American and Hispanic girls and women in research. Public Health Nurs 2013;30(2):159-66.

9. McCormick LK, Crawford M, Anderson RH, Gittelsohn J, Kingsley B, Upson D. Recruiting adolescents into qualitative tobacco research studies: experiences and lessons learned. J Sch Health 1999;69(3):95-9.

10. Diviak KR, Wahl SK, O'Keefe JJ, Mermelstein RJ, Flay BR. Recruitment and retention of adolescents in a smoking trajectory study: who participates and lessons learned. Subst Use Misuse 2006;41(2):175-82.

11. Stanford PD, Monte DA, Briggs FM, Flynn PM, Tanney M, Ellenberg JH, et al.;Reaching for Excellence in Adolescent Care and Health Project. Recruitment and retention of adolescent participants in HIV research: findings from the REACH (Reaching for Excellence in Adolescent Care and Health) Project. J Adolesc Health 2003;32(3):192-203.

12. Meyers K, Webb A, Frantz J, Randall M. What does it take to retain substance-abusing adolescents in research protocols? Delineation of effort required, strategies undertaken, costs incurred, and 6-month post-treatment differences by retention difficulty. Drug Alcohol Depend 2003;69(1):73-85.

13. Story M, Sherwood NE, Obarzanek E, Beech BM, Baranowski JC, Thompson NS, et al. Recruitment of African-American pre-adolescent girls into an obesity prevention trial: the GEMS pilot studies. Ethn Dis 2003;13(1,Suppl 1):S78-87.

14. Resnicow K, Yaroch AL, Davis A, Wang DT, Carter S, Slaughter L, et al. GO GIRLS!: results from a nutrition and physical activity program for low-income, overweight African American adolescent females. Health Educ Behav 2000; 27(5):616-31.

15. Naar-King S, Ellis D, Kolmodin K, Cunningham P, Jen KL, Saelens B, et al. A randomized pilot study of multisystemic therapy targeting obesity in African-American adolescents. J Adolesc Health 2009;45(4):417-9.

16. Embi PJ, Payne PR. Clinical research informatics: challenges, opportunities and definition for an emerging domain. J Am Med Inform Assoc 2009;16(3):316-27.

17. American Medical Informatics Association (AMIA). http:// www.amia.org/. Accessed August 5, 2014.

The opinions expressed by authors contributing to this journal do not necessarily reflect the opinions of the U.S. Department of Health and Human Services, the Public Health Service, the Centers for Disease Control and Prevention, or the authors' affiliated institutions. 
18. Murphy SA, Lynch KG, Oslin D, McKay JR, TenHave T. Developing adaptive treatment strategies in substance abuse research. Drug Alcohol Depend 2007;88(Suppl 2):S24-30.

19. Naar-King S, Ellis D, Idalski Carcone A, Templin T, JacquesTiura AJ, Brogan K, et al. Sequential multiple assignment randomized trial (SMART) to construct weight loss interventions for African American adolescents. J Clin Child Adolesc PsycholForthcoming.

20. Collins LM, Murphy SA, Strecher V. The multiphase optimization strategy (MOST) and the sequential multiple assignment randomized trial (SMART): new methods for more potent eHealth interventions. Am J Prev Med 2007; 32(5Suppl):S112-8.

21. Freed LH, Ellen JM, Irwin CEJr, Millstein SG. Determinants of adolescents' satisfaction with health care providers and intentions to keep follow-up appointments. J Adolesc Health 1998;22(6):475-9.

22. Sawyer SM, Zalan A, Bond LM. Telephone reminders improve adolescent clinic attendance: a randomized controlled trial. J Paediatr Child Health 2002;38(1):79-83.

23. Shilling V, Williamson PR, Hickey H, Sowden E, Beresford MW, Smyth RL, et al. Communication about children's clinical trials as observed and experienced: qualitative study of parents and practitioners. PLoS ONE 2011;6(7):e21604.

24. Young B, Shilling V, Hickey H, Sowden E, Smyth R, Williamson $\mathrm{P}$. What parents think about being approached about children's trials, how this differs from what practitioners expect, and what this tells us about enhancing recruitment. Trials 2011;12(Suppl 1):A116.

25. Keyzer JF, Melnikow J, Kuppermann M, Birch S, Kuenneth C, Nuovo J, et al. Recruitment strategies for minority participation: challenges and cost lessons from the POWER interview. Ethn Dis 2005;15(3):395-406.

26. Rukstalis M, Hauer C. C-B5-05: Early childhood obesity prevention in primary care: opt-in versus opt-out recruitment strategies. Clin Med Res 2011;9(3-4):163-4.

27. Fouad MN, Corbie-Smith G, Curb D, Howard BV, Mouton C, Simon M, et al. Special populations recruitment for the Women's Health Initiative: successes and limitations. Control Clin Trials 2004;25(4):335-52.

28. Carroll JK, Yancey AK, Spring B, Figueroa-Moseley C, Mohr DC, Mustian KM, et al. What are successful recruitment and retention strategies for underserved populations? Examining physical activity interventions in primary care and community settings. Transl Behav Med 2011;1(2):234-51.
29. Resnicow K, Taylor R, Baskin M, McCarty F. Results of Go Girls: a weight control program for overweight AfricanAmerican adolescent females. Obes Res 2005;13(10):1739-48.

30. Story M, Sherwood NE, Himes JH, Davis M, Jacobs DRJr, Cartwright $\mathrm{Y}$, et al. An after-school obesity prevention program for African-American girls: the Minnesota GEMS pilot study. Ethn Dis 2003;13(1,Suppl 1):S54-64.

\footnotetext{
The opinions expressed by authors contributing to this journal do not necessarily reflect the opinions of the U.S. Department of Health and Human Services, the Public Health Service, the Centers for Disease Control and Prevention, or the authors' affiliated institutions.
} 


\section{Tables}

Table 1. Potential Participants in a Weight-Loss Intervention for Obese Racial/Ethnic Minority Adolescents, by Recruitment Strategy, Michigan, 2010-2014

\begin{tabular}{|c|c|c|c|c|}
\hline Component & Informatics & Clinics & Community & Total \\
\hline Potential participants identified, by strategy, $n$ & 643 & 336 & 57 & 1,036 \\
\hline \multicolumn{5}{|l|}{ Screening of EMRs ${ }^{a}, n$} \\
\hline Ineligible individuals & 254 & 68 & 8 & 330 \\
\hline Eligible individuals & 387 & 243 & 49 & 679 \\
\hline
\end{tabular}

Other reasons for ineligibility or nonparticipation during EMR screening, $n$

Ineligible because of participation in earlier study ${ }^{b}$

Refused after receiving opt-out letter ${ }^{c}$

\begin{tabular}{|r|r|r|r|}
\hline 2 & 25 & 0 & 27 \\
\hline 0 & $\mathrm{NA}$ & $\mathrm{NA}$ & 0 \\
\hline
\end{tabular}

Screening by telephone, $\mathrm{n}$

Passive refusal or lost contact

Telephone screens completed

Active refusal

Ineligible

Eligible

\begin{tabular}{|r|r|r|r|}
\hline 268 & 73 & 12 & 353 \\
\hline 119 & 170 & 37 & 326 \\
\hline 15 & 14 & 1 & 30 \\
\hline 25 & 16 & 6 & 47 \\
79 & 140 & 30 & 249 \\
\hline
\end{tabular}

Screening in the home, $\mathrm{n}$

Passive refusal or lost contact

Home screens completed

Active refusal

Ineligible

Eligible and consented

Consented but not enrolled, $n$

\begin{tabular}{|r|r|r|r|}
\hline 18 & 15 & 5 & 38 \\
\hline 61 & 125 & 25 & 211 \\
\hline 0 & 0 & 0 & 0 \\
\hline 4 & 8 & 2 & 14 \\
\hline 57 & 117 & 23 & 197 \\
\hline 4 & 7 & 0 & 11 \\
\hline
\end{tabular}

Family enrollment

Families enrolled, no. $(\% \mathrm{~d})$

Overall enrollment rate, ${ }^{\mathrm{e}}$

Active refusal rate, $\%^{f}$

Passive refusal rate, \%g

Modified active refusal rate, $\%^{\mathrm{h}}$

Modified passive refusal rate, $\%$

119 119

\begin{tabular}{l|l} 
& \\
\hline & \\
\hline & \\
\hline &
\end{tabular}

\begin{tabular}{|l|r|r|r|r|}
\hline & $53(28.5)$ & $110(59.1)$ & $23(12.4)$ & $186(100)$ \\
\hline & 8.2 & 32.7 & 40.4 & 17.8 \\
\hline$\%^{\mathrm{h}}$ & 2.3 & 4.2 & 1.8 & 2.9 \\
\hline$\%^{\mathrm{i}}$ & 45.1 & 28.3 & 29.8 & 39.8 \\
\hline
\end{tabular}

Abbreviations: EMR, electronic medical record; NA, not applicable.

a Screening of EMRs was done for families identified through the informatics strategy, the clinic strategy, and when applicable, the community strategy.

${ }^{b}$ People who participated in an earlier study or whose siblings participated in an earlier study were deemed ineligible.

${ }^{\mathrm{c}}$ The opt-out letter was sent only to families identified through the informatics strategy.

d Percentage of families enrolled, by recruitment strategy.

e Number of enrolled families divided by the number originally identified.

${ }^{f}$ Number of active refusals divided by the number originally identified.

$g$ Number of passive refusals or lost contacts divided by the number originally identified.

${ }^{\mathrm{h}}$ Number of active refusals divided by number deemed eligible by EMR screening.

i Number of passive refusals or lost contacts divided by number deemed eligible by EMR screening.

The opinions expressed by authors contributing to this journal do not necessarily reflect the opinions of the U.S. Department of Health and Human Services, the Public Health Service, the Centers for Disease Control and Prevention, or the authors' affiliated institutions. 
Table 2. Retention Rates in a Weight-Loss Intervention for Obese Racial/Ethnic Minority Adolescents ( $\mathrm{N}=181)$, by Recruitment Strategy, Michigan, 2010-2014

\begin{tabular}{|l|r|r|r|r|}
\hline Strategy & Baseline, No. & 3 Months, No. (\%) & 7 Months, No. (\%) & 9 Months, No. (\%) \\
\hline Informatics & 52 & $49(94.2)$ & $48(92.3)$ & 48 (92.3) \\
\hline Clinics & 107 & $95(88.8)$ & $91(85.0)$ & 20 (90.9) \\
\hline Community & 22 & $21(95.5)$ & $18(86.9)$ \\
\hline Overall & 181 & $165(91.2)$ & $159(87.8)$ & 159 (87.8) \\
\hline
\end{tabular}


Table 3. Baseline Characteristics of Participants $(N=181)$ in a Weight-Loss Intervention for Obese Racial/Ethnic Minority Adolescents, Recruitment Strategy, Michigan, 2010-2014a

\begin{tabular}{|c|c|c|c|c|}
\hline \multirow[b]{2}{*}{ Characteristic } & \multicolumn{3}{|c|}{ Strategy } & \multirow[b]{2}{*}{ Omnibus Test ( $P$ Value) } \\
\hline & Informatics & Clinics & Community & \\
\hline BMI of adolescent, $\mathrm{kg} / \mathrm{m}^{2}$ & $37.8(7.7)$ & $37.6(7.0)$ & $41.5(8.4)$ & $F_{2,178}=2.62(.08)$ \\
\hline $\mathrm{BMI}$ of caregiver, $\mathrm{kg} / \mathrm{m}^{2}$ & $43.5(11.8)$ & $40.4(9.3)$ & $37.7(9.2)$ & $F_{2,176}=3.46(.05)$ \\
\hline Age of adolescent, $y$ & $13.7(1.2)$ & $13.8(1.4)$ & $13.4(1.4)$ & $F_{2,178}=0.81(.45)$ \\
\hline Percentage of adolescents with at least 1 comorbidity & 57.7 & 49.5 & 31.8 & $x_{2}^{2}=4.14(.13)$ \\
\hline Education level of caregiver ${ }^{b}$ & $5.4(1.4)$ & $5.3(1.3)$ & $5.6(1.7)$ & $F_{2,178}=0.47(.62)$ \\
\hline Annual income of caregiver ${ }^{c}$ & $3.7(2.1)$ & $3.2(1.8)$ & $3.4(2.6)$ & $F_{2,175}=0.79(.45)$ \\
\hline
\end{tabular}

Abbreviation: BMI, body mass index.

${ }^{\text {a }}$ All values are mean (standard deviation) unless otherwise indicated.

${ }^{\mathrm{b}}$ Response scale for caregiver education level: 1, did not finish elementary school; 2, finished middle school; 3, finished some high school; 4, high school graduate or general educational development (GED); 5, vocational or training school after high school; 6, some college or associate degree; 7, college graduate or baccalaureate degree; 8 , master's or doctoral degree.

${ }^{\mathrm{C}}$ Response scale for caregiver income: $1,<\$ 5,000 ; 2, \$ 5,000-\$ 11,999 ; 3, \$ 12,000-\$ 15,999 ; 4, \$ 16,000-\$ 24,999 ; 5, \$ 25,000-\$ 34,999 ; 6$,

$\$ 35,000-\$ 49,999 ; 7, \$ 50,000-\$ 74,999 ; 8, \$ 75,000-\$ 99,999 ; 9, \geq \$ 100,000$.

The opinions expressed by authors contributing to this journal do not necessarily reflect the opinions of the U.S. Department of Health and Human Services, the Public Health Service, the Centers for Disease Control and Prevention, or the authors' affiliated institutions. 